\title{
Prevalence and phylogenetic analysis of gastrointestinal helminths (Nematoda: Trichostrongylidae) in ruminant livestock of northwest Iran
}

\author{
Tahereh BARGHANDAN ${ }^{1, a}$, Elham HAJIALILO ${ }^{2,3, b, \bowtie}$, Meysam SHARIFDINI ${ }^{2,4, c, * 凶}{ }^{,}$Amir JAVADI ${ }^{5, d}$ \\ ${ }^{1}$ Qazvin University of Medical Sciences, Student Research Committee; ${ }^{2}$ Qazvin University of Medical Sciences, Medical \\ Microbiology Research Center; ${ }^{3}$ Qazvin University of Medical Sciences, Department of Parasitology and Mycology, Qazvin; \\ ${ }^{4}$ Guilan University of Medical Sciences, School of Medicine, Department of Medical Parasitology and Mycology, Rasht; \\ ${ }^{5}$ Qazvin University of Medical Sciences, Department of Social Sciences, School of Medicine, Qazvin, Iran. \\ aORCID: 0000-0002-4024-0784; ' $O R C I D:$ 0000-0003-2159-4066; ' ORCID: 0000-0003-2686-7748; ' ORCID: 0000-0001-7715-4583
}

${ }^{\square}$ Corresponding author: e.hajialilo@qums.ac.ir, ${ }^{\star} \bowtie$ Corresponding author: sharifdini5@ gmail.com

Received date: 08.07.2019- Accepted date: 21.10.2019

\begin{abstract}
Trichostrongylidae family is considered as a group of gastrointestinal nematodes of ruminants with widespread distribution in the world. The parasites are the major causes of productivity loss in many countries including Iran. This study was carried out to determine the distribution of abomasal Trichostrongylidae among livestock of Qazvin, Iran. Totally, 160 abomasum samples including 83 from sheep, 72 from cattle and 5 from goats were collected from slaughterhouses throughout the Qazvin Province. The ribosomal DNA internal transcribed spacer 2 (ITS2) region was amplified using PCR followed by sequencing 13 different isolates. Phylogenetic analysis of the identified sequences was performed using MEGA 7 software. The prevalence rate of the nematodes was 19.4\% among the specimens. Also, two spices including Marshallagia marshalli and Teladorsagia circumcincta were identified among the isolates with M. marshalli at higher frequency. No Trichostrongylus spp. was detected in the region. Since livestock plays a major role in ecosystem balance and as some species of nematodes have a zoonotic nature, it is necessary to proceed with more comprehensive epidemiologic studies to clarify the infection rate among the human population living in the region.
\end{abstract}

Keywords: Iran, ITS2-rDNA region, Phylogenetic analysis, Qazvin, Trichostrongylidae.

\section{Introduction}

The family of Trichostrongylidae is considered as the most important gastrointestinal parasites among sylvatic and domesticated ruminants with worldwide distribution $(19,38)$. Several species of the family were reported such as Teladorsagia circumcincta, Marshallagia marshalli and Trichostrongylus spp. $(19,33,34,38)$.

Human infection by Trichostrongylus spp. could occur by ingesting the infective-stage larvae or through skin penetration by larvae (14). Human infections are frequently free of apparent clinical symptoms, although gastrointestinal signs may occur in some patients $(31,39)$. The importance of veterinary medicine is not seriously taken into account and this has caused reduced production, economic losses, and public health concerns. These nematodes are considered as major causes of veterinary and zoonotic infections $(12,32)$. Differentiation and improved diagnosis between the species are necessary for control programs as effective control strategies can lead to positive effect over the economy of different countries (3).
Conventional methods based on morphological characters such as spicules, gubernaculum, and caudal bursa in male worms are reliable to detect the species nevertheless, these methods cannot discern the species of female worms $(12,31)$.

Molecular biology assays are applied for identification and phylogenetic analysis of different species of nematodes (38). Although several ribosomal and mitochondrial regions were used to survey the nematodes, investigating the ribosomal DNA sequencing (particularly the ITS2 region) has been shown to be a useful tool for this purpose as the ITS-2 gene region can clarify the intraspecific homogeneity and interspecific divergence of sequence in the species of gastrointestinal nematodes $(5,20,31,36)$.

It is well documented that Iranian researchers have a long term experience over examining the gastrointestinal nematodes among humans and animals by morphological features $(2,7,11)$. Domestically, recent molecular analysis studies are limited to few investigations concerning the diagnosis of Trichostrongylus species, 
without any data available for other species of the Trichostrongylidae family in the country $(13,30,31)$. Due to lack of data on nematodes in the study region, the present research aimed at analyzing the morphological features as well as molecular characterization of ITS2 region of different species of the helminthes.

\section{Material and Methods}

Sample collection and specimens examined: Totally, 160 abomasum samples were collected from 83 sheep, 72 cattle and 5 goats during 2017-2018. The animals originated from Qazvin Province, located in northern margin of central Iran and were slaughtered at two local abattoirs of the cities of Qazvin and Takestan (Figure 1). This study was approved by the Medical Ethics Committee of Qazvin University of Medical Sciences (Approval code: IR.QUMS.REC.1396.375). All specimens were examined for infectivity by Trichostrongylidae family through washing the content of each abomasum by passing through 20, 40 and 100 mesh sieves and observed with stereomicroscope. The helminths were collected and cleaned with normal saline followed by addition of lactophenol to observe their morphological features. The specimens were preserved in $70 \%$ ethanol and kept at room temperature.

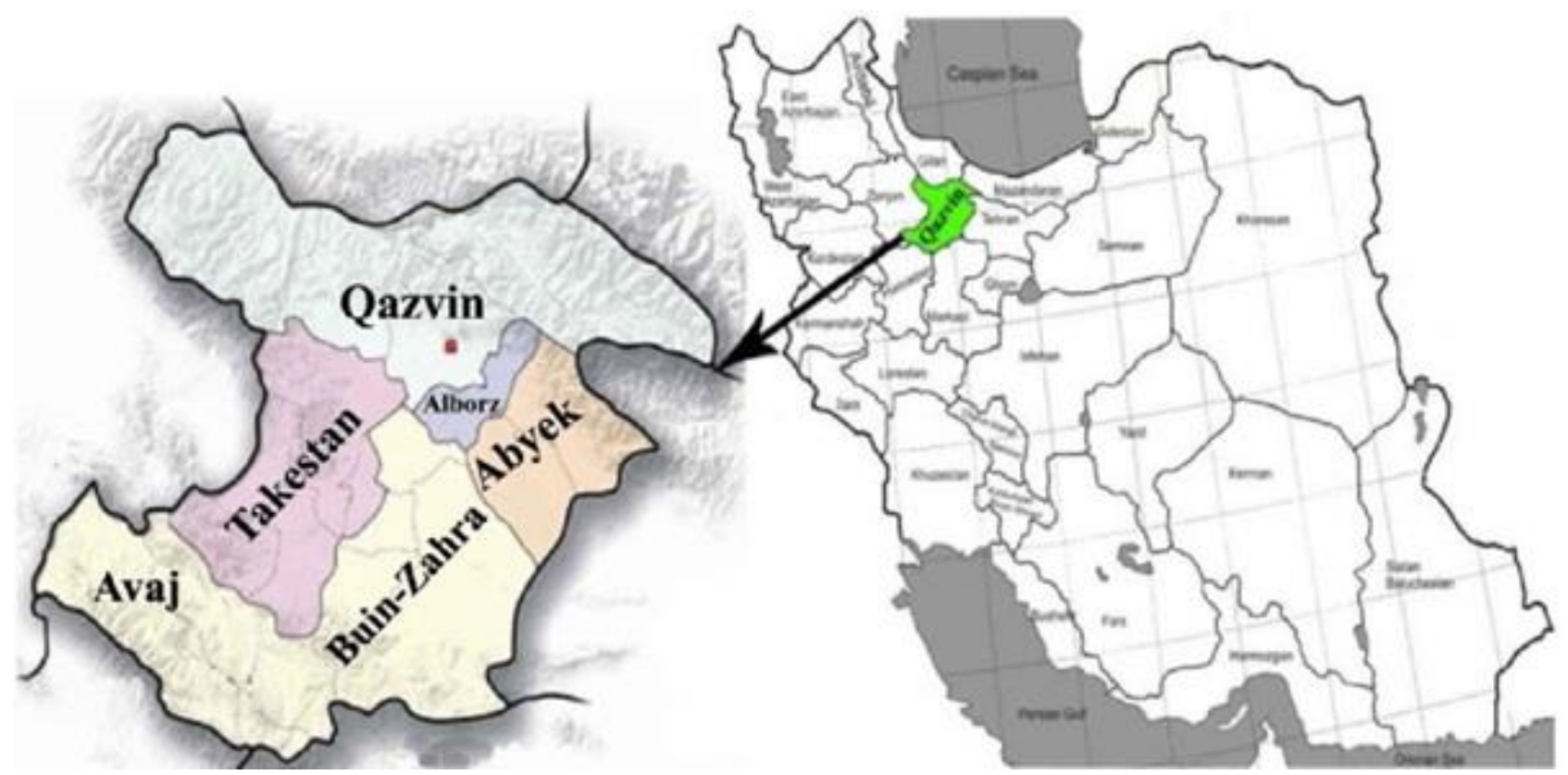

Figure 1. Map of Qazvin Province, located in the northern margin of central Iran.

DNA extraction and PCR assay: Twenty female specimens were randomly selected for DNA extraction. The helminth was minced by 6 rounds of freeze-thaw cycles using $200 \mathrm{mg}$ tissue lysis buffer and the finely minced worm was used the High Pure PCR Template Preparation Kit (Roche, Mannheim, Germany) for DNA extraction according to the manufacturer's recommended protocol.

Fragments of ribosomal DNA internal transcribed spacer 2 (ITS2) region with approximately 328 bp was amplified with specific primers (30). Thirty microliter reaction volume containing $15 \mu \mathrm{l}$ of PCR mix including Taq DNA polymerase, dNTPs and $\mathrm{MgCl}_{2}$ (2 x Master Mix RED Ampliqon, Denmark), $11 \mu \mathrm{l}$ of molecular biology grade water, $1 \mu \mathrm{l}$ of each primer $(10 \mathrm{pmol} / \mu \mathrm{l})$ and $2 \mu \mathrm{l}$ of DNA template was applied for each reaction. The PCRs were included the following temperatures, $94^{\circ} \mathrm{C}$ for $20 \mathrm{~s}$ (denaturation), $55^{\circ} \mathrm{C}$ for $20 \mathrm{~s}$ (annealing), $72^{\circ} \mathrm{C}$ for $30 \mathrm{~s}$ (extension) for 35 cycles and a final extension at $72^{\circ} \mathrm{C}$ for 5 min.

Sequencing and phylogenetic analysis: Following the purification (AccuPrep ${ }^{\circledR}$ PCR/Gel Purification KitBioneer, Korea) of PCR products, each specimen was sequenced using Applied Biosystems ABI 3730xl DNA analyzer sequencing (Bioneer, Korea). The nucleotide sequences were compared with each other and with reference sequences using BioEdit software and adjusted manually (18). Multiple sequence alignment was made with the ClustalW method and further compared with the sequences present in the GenBank sequence database. Phylogenetic tree was constructed using Tamura 3parameter model of ML (Maximum likelihood) method by the Molecular \& Evolution Genetic Analysis software version 7 (MEGA 7.0). Bootstrap value was considered based on 1000 replications. Necator americanus sequence was considered as outgroup (Figure 2). 


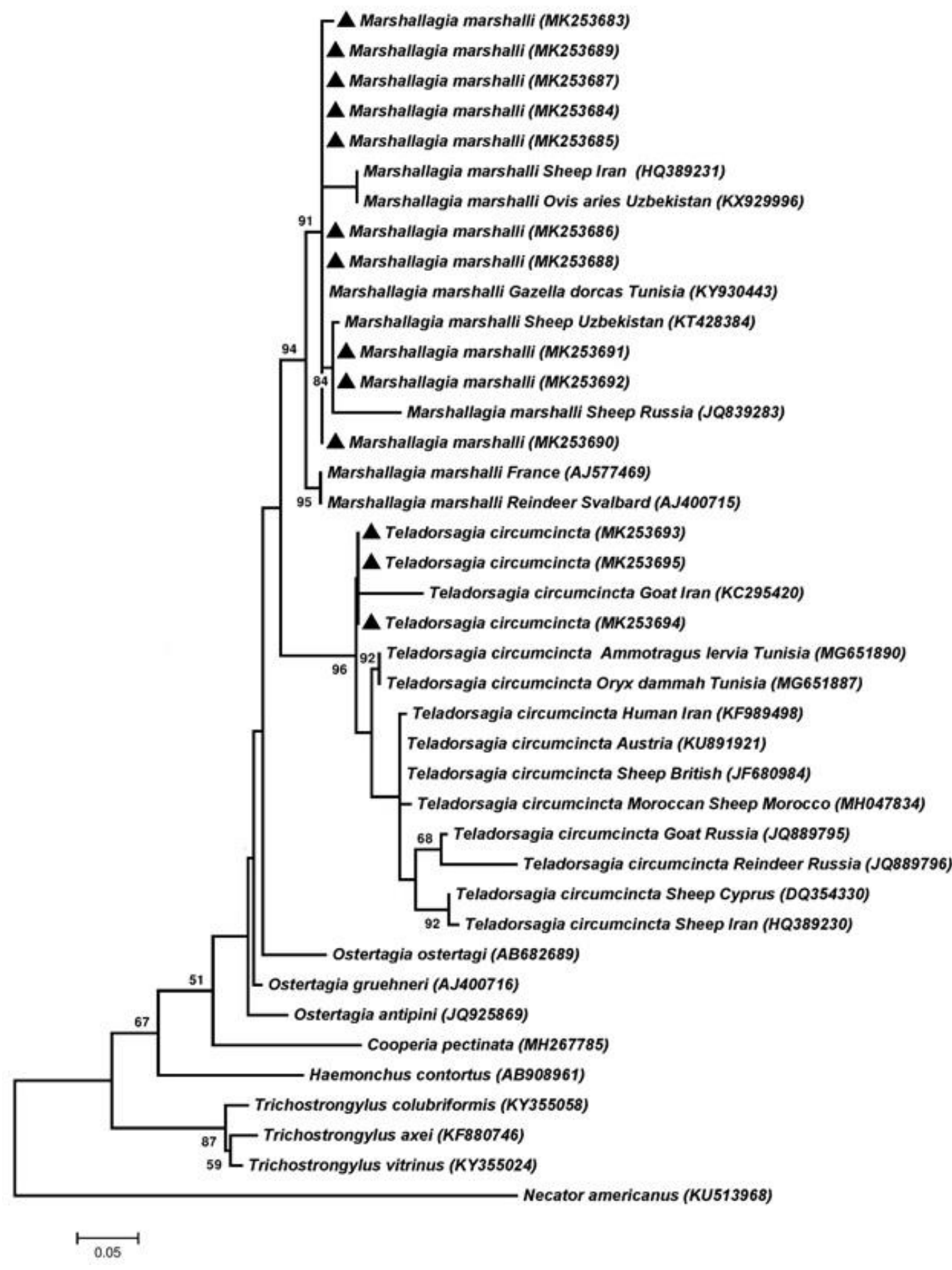

Figure 2. Phylogenetic tree of Trichostrongylidae family obtained in this study $(\boldsymbol{\Delta})$ and reference sequences retrieved from GenBank based on ITS2 nucleotide sequences and constructed using the Tamura 3-parameter model in MEGA7 software. Necator americanus considered as an out group.

\section{Results}

Out of 160 specimens collected from sheep $(51 \%)$, cattle $(45 \%)$, and goats $(3 \%)$ in the present study, $19.4 \%$ (31/160) samples were found to be infected with Trichostrongylidae. The prevalences of nematodes were $33.7 \%(28 / 83)$ and $60 \%(3 / 5)$ among sheep and goat, respectively. No infection was found in cattle specimens. Two species including Marshallagia marshalli and Teladorsagia circumcincta were identified among the isolates by morphological features which was later confirmed by molecular analysis (Figure 3 ). Infection rate of $M$. marshalli and T. circumcincta among sheep and goats are clarified in Table 1. None of Trichostrongylus spp. was detected in the study. A total of 13 ITS2sequences of nematodes were deposited in GenBank, including ten $M$. marshalli (accession numbers: MK253683-MK253692) and three $T$. circumcincta (accession numbers: MK253693-MK253695). In addition, 

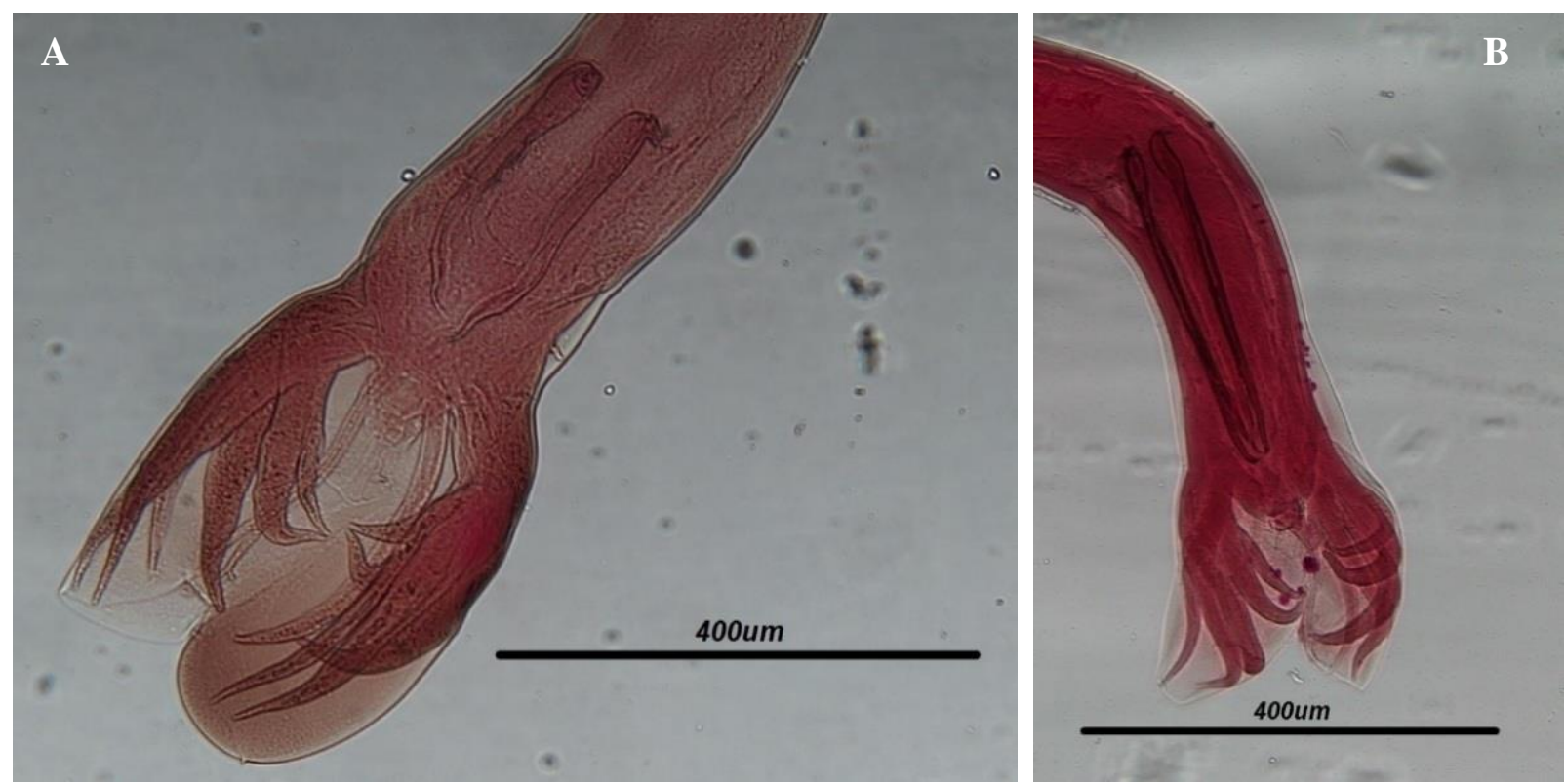

Figure 3. A: Copulatory bursa and spicules of Marshallagia marshalli; B: Teladorsagia circumcincta.

the molecular survey demonstrated high similarity (more than $95 \%$ ) between the sequences found in our study and those of M. marshalli and $T$. circumcincta in the GenBank sequence database.

Table 1. Trichostrongylidae family species isolated from livestock in Qazvin, Iran.

\begin{tabular}{cccc}
\hline \multirow{2}{*}{ Host } & \multicolumn{2}{c}{ Number of male worms in abomasum } & \\
\cline { 2 - 3 } & $\begin{array}{c}\text { T. circumcincta } \\
(n / \%)\end{array}$ & $\begin{array}{c}\text { M. marshalli } \\
(n / \%)\end{array}$ & Total \\
\hline Sheep & $5(6)$ & $78(93)$ & 83 \\
Goat & $5(25)$ & $15(75)$ & 20 \\
\hline Total & $10(9.7)$ & $93(90)$ & 103 \\
\hline
\end{tabular}

A dendrogram based on the phylogenetic analysis revealed that morphological examination can detect the different species of the parasite (Figure 2). The isolates were grouped into two distinct clusters corresponding to the M. marshalli and $T$. circumcincta alongside the gene references. Multiple alignments represented that the intraspecies similarity for $M$. marshalli and T. circumcincta were $98-100 \%$ and $100 \%$, respectively.

\section{Discussion and Conclusion}

Trichostrongylidae family is a group of significant parasites among sylvatic and domesticated ruminants and this makes the nematode infection as a common disease between the human and ruminant animals in endemic regions. Contamination by Trichostrongylidae nematodes can cause permanent damage to livestock industry, leading to changes in animal body weight and decreased production output. The most frequent species of the family in ruminant are Nematodirus, Marshallagia, Haemonchus, Cooperia, Trichostrongylus and Teladorsagia (4, 6, 10, 21, 28).

The present study is the first report of the nematodes in Qazvin, Iran. This research revealed that the infection rate among sheep and goats were $33.7 \%$ and $60 \%$, respectively. Several studies reported from Iran showed higher infection rate in livestock compared to that of the current research. Eslami et al. (7) reported an infection rate as $88 \%$ in sheep. Also, Borji et al. (2) described the presence of high infection rate $(75.1 \%)$ by gastrointestinal helminths in camels. Mashayekhi et al. (25) found an infection rate equal to $44 \%$ among the abomasal nematodes of cattle. Unlike the research stated above, few studies have been reported lower infection rate in livestock $(8,27)$.

Based on morphological and molecular analysis, two species including $M$. marshalli and T. circumcincta were found among the domestic ruminants in Qazvin Province, Iran. Marshallagia marshalli was the most frequent species $(90 \%)$, while $T$. circumcincta $(9.7 \%)$ was less prevalent among the isolates. Aligned with our findings, the most commonly reported species among the livestock in different parts of the country was M. marshalli as claimed by Eslami et al. (7) who found M. marshalli (93\%) and T. circumcincta (9.3\%) among the wild sheep (Ovis orientalis). Borji et al. (2) has also reported $M$. marshalli and $T$. circumcincta in camel isolates in the northeast of Iran. Other studies carried out in different parts of Iran (including the southeastern, northwest, and central parts) determined isolation of the parasite species from sheep and goat $(17,23,28,35)$. Conversely, few 
studies have shown the higher frequency of $T$. circumcincta than $M$. marshalli in the ruminants. Nabavi et al. (27) found $T$. circumcincta at higher prevalence (19.3\%) than M. marshalli $(12.2 \%)$ in sheep isolates in the northern, central, and southern regions of Iran (Mazanderan, Isfahan and Khuzestan Provinces). Several studies have revealed the presence of M. marshalli and $T$. circumcincta among the ruminants in different parts of the world $(5,20,26,37)$.

Considering the extensive use of anti-parasitic drugs in the country, the main cause of high prevalence rate for nematodes is related to drug resistance emerged by the worms or might be due to decreased host immune response (15). The government strategic treatment of local livestock started since 1968 by administration of albendazole, twice a year for a duration of years (8), and subsequently, several species including $M$. marshalli, $T$. circumcincta, $T$. colubriformis, and $H$. contortus exhibited resistance to therapy $(15,28)$.

In domestic traditional animal husbandry throughout the country, the flocks of sheep and goats are kept and grazed together and this causes the persistence of the parasites in both types of farm animals (28), and the results of our study confirmed the presence of both M. marshalli and $T$. circumcincta species in two different groups of animals. It is documented that goats acquire a lower level of immunity against the parasites compared to sheep helminths of ruminants, hence goats experience more severe disease (16), however, the number of goat specimens used in the current research was limited and no further useful suggestion could be made on infection rate among the sheep and goat isolates in the study areas.

The family of Trichostrongylidae is classified as gastrointestinal helminths of ruminants, however, few studies have categorized these parasites according to their infection in human. The recovery of Haemonchus contortus, Ostertagia ostertagi, and Marshallagia marshalli were reported from human cases in Iran (9). Therefore coexistence of human alongside the ruminant animals could lead to human infection.

Iran is considered as a major focus for human and animal trichostrongyliasis in the world $(10,13,30,31)$ and several species of Trichostrongylus were reported from different parts of the country including T. colubriformis, T. vitrinus, T. axei, T. capricola, T. probolurus, $T$. longispicularis, T. orientalis, T. lerouxi, T. skrjabini, and T. hamatus $(1,10,12,31)$.

A striking finding of the present research was the lack of Trichostrongylus spp. and consistent with our results several studies failed to observe these nematodes among the specimens across the country $(8,17,23,27)$. The nematodes distribution in different geographical regions is depending on climate condition, pasture type, and management (29). It is obvious that the favorable conditions helps the existence of the nematode, therefore the absence of these parasites may be due to the drug sensitivity demonstrated by Trichostrongylus spp.

In the current study, the ITS2 region of rDNA was chosen for detecting the species of Trichostrongylidae family as several studies had used the region for molecular analysis $(5,13,22,24,30,38)$. The phylogenetic analysis performed for the present study clarified that all $M$. marshalli and $T$. circumcincta isolates were placed in one branch together with other specimens of different parts of the world. BLAST analysis indicated that there was $100 \%$ homology between the MK253683, MK253689, MK253687, MK253685, MK253686, MK253690, and MK253688 from sheep M. marshalli isolates and the MK253684 recovered from goats and that these isolates also exhibited $100 \%$ similarity to $M$. marshalli sequence (KY930443) from Gazella dorcas in Tunisia. In addition, there was $100 \%$ homology between the MK253691 and MK253692 obtained from the goat and sheep isolates, respectively. These isolates were grouped with sheep isolates from Uzbekistan (KT428384) and Russia (JQ839283). Also, there was 100\% homology between the T. circumcincta isolates from sheep (MK253695) and those isolated from goats (MK253693 and MK253694). These isolates were grouped with goat (KC295420) isolate from Iran.

In conclusion, the present study demonstrated two species including $M$. marshalli and $T$. circumcincta belonging to the Trichostrongylidae family among the goats and sheep in the study region. In addition, it showed that M. marshalli is the predominant species among the local ruminants. The prevalence of nematodes could be related to the geographical condition, ecology, and the use of antiparasitic drugs.

\section{Acknowledgements}

The authors are grateful to the abattoir personnel in Qazvin Province. We would like to appreciate the assistance offered by the colleagues at Department of Parasitology and Mycology, Medical School, Qazvin University of Medical Sciences. The study was financially supported by a grant from Qazvin University of Medical Sciences (Project No: 888). We thank Dr. Ali Asghar Pahlevan for editing the final version of the English manuscript.

\section{Conflict of Interest}

The authors declared that there is no conflict of interests.

\section{References}

1. Anvari-Tafti M, Sazmand A, Hekmatimoghaddam S, et al (2013): Gastrointestinal helminths of camels (Camelus dromedarius) in center of Iran. Trop Biomed, 30, 56-61. 
2. Borji H, Razmi G, Movassaghi AR, et al (2010): A study on gastrointestinal helminths of camels in Mashhad abattoir, Iran. Iran J Vet Res, 11, 174-179.

3. Bott NJ, Campbell BE, Beveridge I, et al (2009): $A$ combined microscopic-molecular method for the diagnosis of strongylid infections in sheep. Int J Parasitol, 39, 12771287.

4. Bradbury R (2006): An imported case of Trichostrongylid infection in Tasmania \& A review of human Trichostrongylidiosis. Annals of the ACTM, 7, 25-28.

5. Dallas J, Irvine R, Halvorsen O, et al (2000): Identification by polymerase chain reaction (PCR) of Marshallagia marshalli and Ostertagia gruehneri from Svalbard reindeer. Int J Parasitol, 30, 863-866.

6. Dróżdż J, Derniaszkiewicz AW, Lachowicz J (2002): Exchange of gastrointestinal nematodes between roe and red deer (Cervidae) and European bison (Bovidae) in the Bieszczady Mountalns (Garpathians, Poland). Acta Parasitol, 47, 314-317.

7. Eslami A, Meydani M, Maleki S, et al (1979): Gastrointestinal nematodes of wild sheep (Ovis orientalis) from Iran. J Wildl Dis, 15, 263-265.

8. Garedaghi Y, Hashemzadefarhang H, Fattahi A (2013): Prevalence of abomasal nematodes in sheep slaughtered at Baneh town. Am J Anim Vet Sci, 8, 142-145.

9. Ghadirian E, Arfaa FNMN(1973): First report of human infection with Haemonchus contortus, Ostertagia ostertagi, and Marshallagia marshalli (family Trichostrongylidae) in Iran. J Parasitol, 59, 1144-1145.

10. Ghadirian E, Arfaa F (1975): Present status of trichostrongyliasis in Iran. Am J Trop Med Hyg, 24, 935941.

11. Ghadirian E, Arfaa F, Sadighian A (1974): Human infection with Trichostrongylus capricola in Iran. Am J Trop Med Hyg, 23, 1002-1003.

12. Ghasemikhah R, Mirhendi H, Kia E, et al (2011): Morphological and morphometrical description of Trichostrongylus species isolated from domestic ruminants in Khuzestan Province, southwest Iran. Iran J Parasitol, 6, 82-88.

13. Ghasemikhah R, Sharbatkhori M, Mobedi I, et al (2012): Sequence analysis of the second internal transcribed spacer (ITS2) region of rDNA for species identification of Trichostrongylus nematodes isolated from domestic livestock in Iran. Iran J Parasitol, 7, 40-46.

14. Gholami S, Babamahmoodi F, Abedian R, et al (2015): Trichostrongylus colubriformis: possible most common cause of human infection in Mazandaran Province, North of Iran. Iran J Parasitol, 10, 110-115.

15. Gholamian A, Eslami A, Nabavi, L, et al (2007): A field survey on resistance to albendazole in gastrointestinal nematodes of sheep in Khuzestan Province of Iran. J Vet Res, 62, 45-51.

16. Gorski P, Niznikowski R, Strzelec E, et al (2004): Prevalence of protozoan and helminth internal parasite infections in goat and sheep flocks in Poland. Arch Tierz, 47, 43-49.

17. Hakimzadegan M, Khosroshahi MK (2013): Prevalence of abomasal nematodes in slaughtered goats at industrial
Urmia slaughterhouse, West Azerbaijan Province, Northwest of Iran. J Anim Poultry Sci, 2, 120-124.

18. Hall TA (1999): BioEdit: a user-friendly biological sequence alignment editor and analysis program for Windows 95/98/NT. Nucleic Acids Symp Ser, 41, 95-98.

19. Hoberg EP, Lichtenfels JR (1994): Phylogenetic systematic analysis of the Trichostrongylidae (Nematoda), with an initial assessment of coevolution and biogeography. J Parasitol, 80, 976-996.

20. Hoberg EP, Monsen KJ, Kutz S, et al (1999): Structure, biodiversity, and historical biogeography of nematode faunas in holarctic ruminants: morphological and molecular diagnoses for Teladorsagia boreoarcticus $n$. sp.(Nematoda: Ostertagiinae), a dimorphic cryptic species in muskoxen (Ovibos moschatus). J Parasitol, 85, 910-934.

21. Holmes P (1987): Pathophysiology of nematode infections. Int J Parasitol, 17, 443-451.

22. Hoste H, Chilton NB, Beveridge I, et al (1998): $A$ comparison of the first internal transcribed spacer of ribosomal DNA in seven species of Trichostrongylus (Nematoda: Trichostrongylidae). Int J Parasitol, 28, 12511260.

23. Kordi B, Mirzaei M, Nooshadokht M (2019): Identification of abomasum nematodes fauna of ruminant in Kerman industrial slaughterhouse, Iran. Biomed J Sci \& Tech Res, 20, 14970- 14973.

24. Kuznetsov D, Kuznetsova N (2007): Sequences of the second internal transcribed spacer of ribosomal DNA for three species of Trichostrongylus (Nematoda: Trichostrongylidae) from sheep in Russia. Helminthologia, 44, 43-46.

25. Mashayekhi M, Gharedaghi Y, Farazmand MR (2013): Study of abomasal nematodes in adult cattles in abattoir of Tabriz Iran. Bull Env Pharmacol Life Sci, 2, 107-109.

26. Meradi S, Bentounsi B, Zouyed I, et al (2011): The steppe species of gastrointestinal nematodes of small ruminants, with a focus on Marshallagia: climate as a key determinant. Parasite, 18, 261-269.

27. Nabavi R, Eslami A, Shokrani H, et al (2011): Study on the prevalence, intensity and seasonal dynamics of abomasal helminths in sheep from different climatic zones of Iran. World Appl Sci J, 12, 441-445.

28. Pestechian N, Kalani H, Faridnia R, et al (2014): Zoonotic gastrointestinal nematodes (Trichostrongylidae) from sheep and goat in Isfahan, Iran. Acta Sci Vet, 42, 1-6.

29. Roeber F, Jex AR, Gasser RB (2013): Impact of gastrointestinal parasitic nematodes of sheep, and the role of advanced molecular tools for exploring epidemiology and drug resistance-an Australian perspective. Parasit Vectors, 6, 153.

30. Sharifdini M, Derakhshani S, Alizadeh SA, et al (2017): Molecular identification and phylogenetic analysis of human Trichostrongylus species from an endemic area of Iran. Acta Trop, 176, 293-299.

31. Sharifdini M, Heidari Z, Hesari Z, et al (2017): Molecular phylogenetics of Trichostrongylus species (Nematoda: Trichostrongylidae) from humans of Mazandaran Province, Iran. Korean J Parasitol, 55, 279285. 
32. Steel J, Jones W, Symons L (1982): Effects of a concurrent infection of Trichostrongylus colubriformis on the productivity and physiological and metabolic responses of lambs infected with Ostertagia circumcincta. Aust J Agric Res, 33, 131-40.

33. Sun MM, Han L, Zhang FK, et al (2018): Characterization of the complete mitochondrial genome of Marshallagia marshalli and phylogenetic implications for the superfamily Trichostrongyloidea. Parasitol Res, 117, 307-313.

34. Suarez V, Cabaret J (1991): Similarities between species of the Ostertagiinae (Nematoda: Trichostrongyloidea) in relation to host-specificity and climatic environment. Syst Parasitol, 20, 179-85.

35. Talari SA, Arbabi M (2004): Prevalence of Iarge intestinal nematodes of ruminant in slaughterhouse of Kashan. Feyz J Kashan Univ Med Sci, 8, 45-50.
36. Tan TK, Panchadcharam C, Low VL, et al (2014): Coinfection of Haemonchus contortus and Trichostrongylus spp. among livestock in Malaysia as revealed by amplification and sequencing of the internal transcribed spacer II DNA region. BMC Vet Res, $\mathbf{1 0}, 38$.

37. Tariq KA, Chishti MZ, Ahmad F, et al (2008): Epidemiology of gastrointestinal nematodes of sheep managed under traditional husbandry system in Kashmir valley. Vet Parasitol, 158, 138-143.

38. Von Samson-Himmelstjerna G, Harder A, Schnieder T (2002): Quantitative analysis of ITS2 sequences in trichostrongyle parasites. Int J Parasitol, 32, 1529-1535.

39. Wall EC, Bhatnagar N, Watson J, et al (2010): $A n$ unusual case of hypereosinophilia and abdominal pain: an outbreak of Trichostrongylus imported from New Zealand. J Travel Med, 18, 59-60. 